\title{
A fatal case of necrotizing myositis panniculitis from E.coli in a patient with alcohol cirrhosis and MGUS: Case Report
}

\author{
April Jensen ${ }^{1 *}$ and Nathan Smith ${ }^{2}$ \\ ${ }^{1}$ DO, MPH, Good Samaritan Regional Medical Center, ACOS, Corvallis, OR 97330, USA \\ ${ }^{2}$ DO, ACOS, Good Samaritan Regional Medical Center, ACOS, Corvallis, OR 97330, USA
}

\begin{abstract}
Necrotizing infections are devastating diseases that lead to rapid deterioration with high mortality rates, especially when misdiagnosed or underrecognized. There are three main categories for necrotizing soft tissue infections (NSTI) including necrotizing cellulitis, fasciitis, and myositis. The most severe is necrotizing myositis because it extends beyond the fascia which then requires a much larger debridement for source control. The types of infections include polymicrobial and monomicrobial usually Group A Streptococcus (GAS) pyogenes. We present a case of monomicrobial Escherichia coli (E. coli) necrotizing myositis of a pannus in a patient with alcoholic cirrhosis and monoclonal gammopathy of undetermined significance (MGUS).
\end{abstract}

\section{Case review}

Our patient is a 51-year-old male with history of alcoholic cirrhosis with MELD 24 on admission, hypertension, morbid obesity (BMI 41) and MGUS with chronic thrombocytopenia. He was admitted to the hospital on with complaints of nausea and vomiting for the last three weeks but worsening over the last four days prior to admission and suspected viral vs bacterial gastroenteritis. Abdominal ultrasound on admission showed cirrhosis, portal hypertension, splenomegaly, cholelithiasis and ascites (Figure 1). Over the next 24 hours, he had worsening left lower quadrant abdominal pain, fevers, and increasing diarrhea which prompted a CT scan which showed non-specific findings of cirrhosis with moderate splenomegaly and widespread edema in the left abdominal wall subcutaneous tissue. At this time, it was noted that he had a red patch over his pannus as well as chronic woody edema of his pannus and he was started on Vancomycin \& Ceftriaxone for treatment of panniculitis (Figure 2). Fecal studies were negative for shiga toxin $1 \& 2$, stool cultures $4+$ usual fecal flora, Clostridium difficile negative, and fecal leukocytes negative. Within 48 hours of admission, he became hypotensive, obtunded requiring intubation and was started on sepsis protocol. On hospital day \#4, general surgery was consulted for worsening erythema of his pannus. On repeat labs his WBC was 40, lactate 10.6, CRP 8.86, Na+ 126, Cr 2.6, pH 6.95 and LRINEC (Laboratory Risk Indicators for NECrotizing fasciitis). He was started on broad spectrum antibiotics including Cefepime, Clindamycin, Flagyl, Vancomycin and continued with aggressive fluid resuscitation as well as vasopressors and bicarbonate drip. He was considered too unstable on max doses of three vasopressors including Levophed, Epinephrine \& Vasopressin, so we did not proceed to the OR for possible excisional debridement of his pannus. A bedside diagnostic excisional debridement was performed which showed extensive myonecrosis of his pannus. Given the severity of his disease and his underlying comorbidities, no further debridement was done. It was felt that he was would require an extensive abdominal wall debridement which he was unlikely to survive. The family gathered at the bedside before he was made comfort care and then quickly expired. Cultures from his debridement grew $3+E$. coli as the only organism.

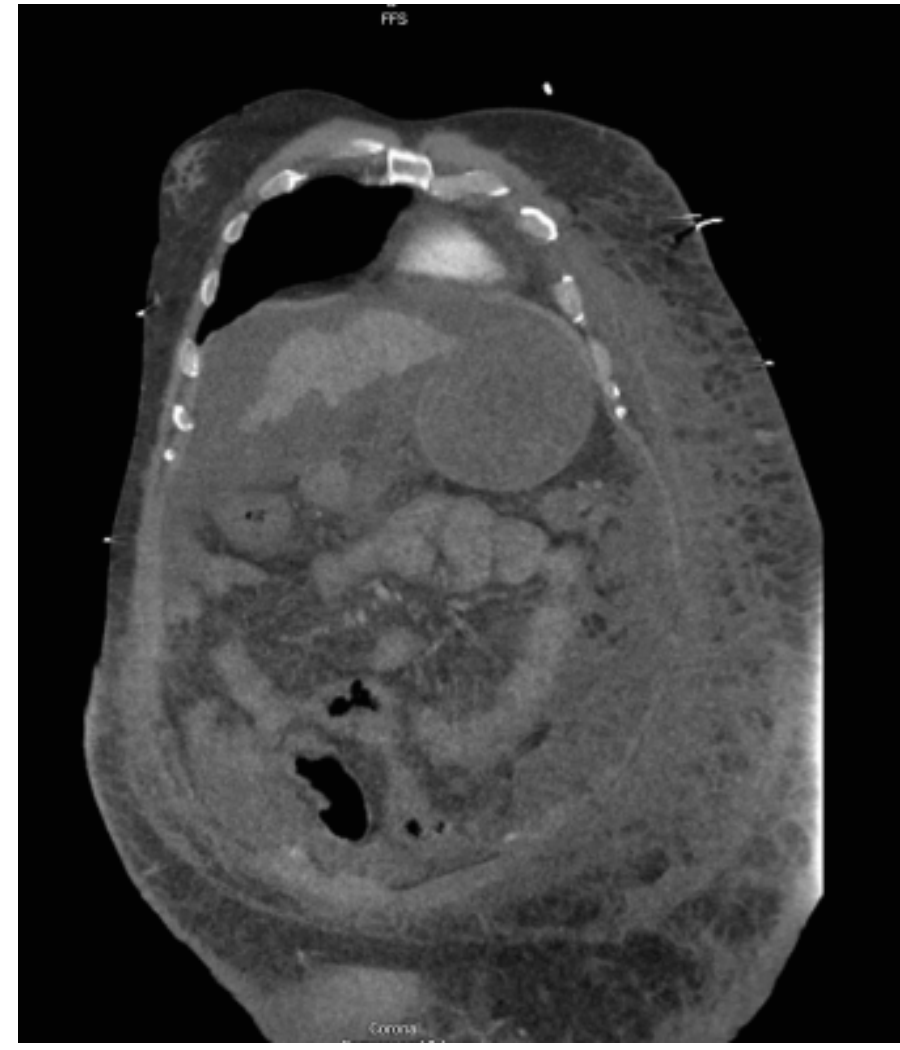

Figure 1. Abdominal CT showing cirrhosis, ascites, splenomegaly with portal hypertension, cholelithiasis and diffuse abdominal wall edema

${ }^{\star}$ Correspondence to: April Jensen, DO, MPH, Good Samaritan Regional Medical Center, ACOS, Corvallis, OR 97330, USA, E-mail: ajensen@samhealth.org

Received: October 23, 2019; Accepted: October 29, 2019; Published: October 31,2019 


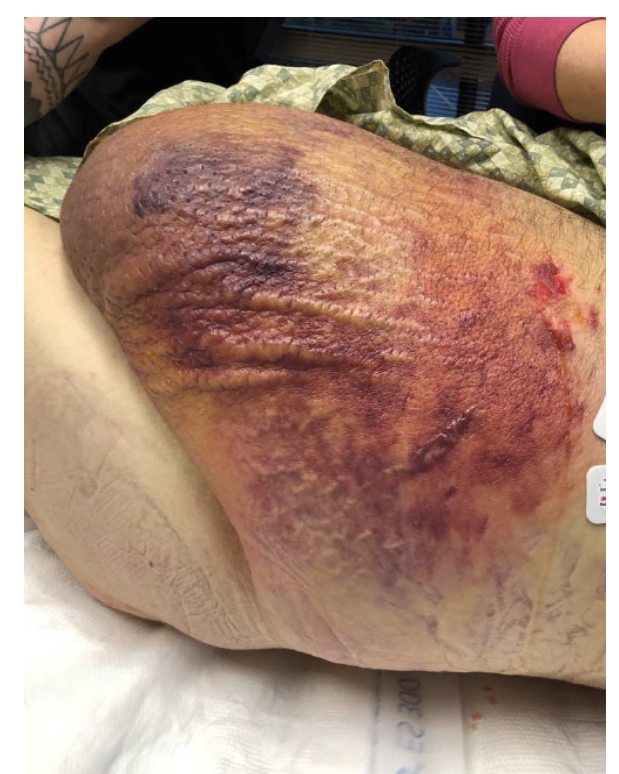

Figure 2. Widespread edema in the left abdominal wall with ecchymosis and skin mottling

\section{Discussion}

Necrotizing infections can be devastating diseases with a high mortality rate. The incidence in the United States is approximately 1,000 per year [1]. Mortality approaches $50 \%$ but non-operative management has an almost $100 \%$ mortality rate in true necrotizing infections. Risk factors include immunocompromised states such as diabetes, cancer, HIV/AIDS, chemotherapy, steroids, or diabetes. Other risk factors include IV drug use. Source control includes local wide debridement and can leave the patient with very morbid surgical wounds. In this patient, he presented with a past medical history of MGUS which adds a unique risk factor to this case and overall can increase bacterial \& viral infection risk by $>2$ fold [2]. His pannus cultures grew monomicrobial organism, E. coli which is also unique presentation of a necrotizing soft tissue infection. There are four subtypes of necrotizing infections including type I- polymicrobial which is usually staph, strep, E. coli, pseudomonas, and anaerobes. Type II- monomicrobial usually group A strep or S. pyogenes. Type III - Vibrio found in saltwater and Type IV- fungal. Gram negative necrotizing infections have been reported as an association with liver cirrhosis and portend a poorer clinical outcome [1]. On initial presentation, his LRINEC score was only 2 but then climbed to 6 at the time of his death. LRINEC can be useful to help differentiate cellulitis from necrotizing infections [3] but it is an outdated model and the validation studies have failed to show the original high specificity and sensitivity [4]. Overall, LRINEC should not be used to exclude the disease because of the high risk of missed diagnosis in these patients [5]. NSTI remains a clinical diagnosis and usually presents with pain out of proportion to the exam, rapidly expanding erythema and edema, fevers and occasionally crepitus, bullae, skin necrosis/ecchymosis as well as signs of sepsis [1]. The definitive treatment is wide local debridement, but you can consider additional therapies such as hyperbaric oxygen and IVIG in certain patient populations [6]. In this patient, he had a very severe fatal disease of myonecrosis in an unusual pannus location with a rare monomicrobial organism E. coli.

\section{References}

1. Goldstein EJ, Anaya DA, Dellinger EP (2007) Necrotizing soft-tissue infection: diagnosis and management. Clin Infect Dis 44: 705-710.

2. Kristinsson SY, Tang M, Pfeiffer RM, Björkholm M, Goldin LR, et al. (2012) Monoclonal gammopathy of undetermined significance and risk of infections: a population-based study. haematologica 97 : 854-858.

3. Neeki MM, Dong F, Au C, Toy J, Khoshab N, et al. (2017) Evaluating the laboratory risk indicator to differentiate cellulitis from necrotizing fasciitis in the emergency department. West J Emerg Med 18: 684-689.

4. Liao CI, Lee YK, Su YC, Chuang CH, Wong CH (2012) Validation of the laboratory risk indicator for necrotizing fasciitis (LRINEC) score for early diagnosis of necrotizing fasciitis. Tzu Chi Med J 24: 73-76.

5. Wong (2004) The LRINEC (labarotry risk indicator of necrotizing fasciitis) score: tool for distinguishing necrotizing fasciitis from other soft tissue infections. Crit Care Med pp: 1535-1541.

6. Park SY, Yu SN, Lee EJ, Kim T, Jeon MH, et al. (2019) Monomicrobial gram-negative necrotizing fasciitis: An uncommon but fatal syndrome. Diagn Microbriol Infect Dis 94: 183-187.

Copyright: (C2019 Jensen A. This is an open-access article distributed under the terms of the Creative Commons Attribution License, which permits unrestricted use, distribution, and reproduction in any medium, provided the original author and source are credited. 